\title{
Evaluation of the root and canal morphology of mandibular permanent molars in a south-eastern Turkish population using cone-beam computed tomography
}

\author{
Bilge Gulsum Nur ${ }^{1}$, Evren $\mathrm{Ok}^{2}$, Mustafa Altunsoy ${ }^{1}$, Osman Sami Aglarci ${ }^{3}$, Mehmet Colak \\ Enes Gungor ${ }^{4}$
}

Correspondence: Dr. Bilge Gulsum Nur Email: dtbilgenur@hotmail.com

\begin{abstract}
'Department of Pedodontics, Faculty of Dentistry, Sifa University, Izmir, Turkiye,

${ }^{2}$ Department of Endodontics, Faculty of Dentistry, Sifa University, Izmir, Turkiye,

${ }^{3}$ Department of Oral and Maxilla Facial Radiology, Faculty of Dentistry, Sifa University, Izmir, Turkiye, ${ }^{4}$ Department of Oral and Maxilla Facial Radiology, Dicle University, Diyarbakir, Turkiye
\end{abstract}

\section{ABSTRACT}

Objective: The aim of this retrospective study was to determine the root and canal morphology of the mandibular first and second permanent molars in a Turkish population using cone-beam computed tomography (CBCT). Materials and Methods: CBCT images of mandibular first $(n=966)$ and second molar $(n=1165)$ teeth from 850 Turkish patients were evaluated. The root canal configurations were classified according to the method of Vertucci. The data were analyzed by Pearson's Chi-square test. Results: The majority of mandibular molars were two rooted with three canals; however, three roots were identified in $0.05 \%$ of the first molars and $0.01 \%$ of the second molars, and $100 \%$ of the additional root canals were of type I configuration. Mesial roots had more complex canal systems with more than one canal, whereas most distal roots had a type I configuration. Conclusions: Within the limitations of this study, it can be concluded that CBCT scanning provides supplemental information about the root canal configurations of mandibular molars in a Turkish population. This study may help clinicians in the root canal treatment of mandibular molars.

Key words: Cone-beam computed tomography, mandibular permanent molars, root and canal morphology, south-eastern Turkish population

\section{INTRODUCTION}

The morphology of the root canal systems of mandibular molars may vary according to ethnic differences and origin, age, gender, and study design. ${ }^{[1-3]}$ The knowledge of morphological characteristics and variations of root canal plays an important role in the success of endodontic diagnosis and treatment. ${ }^{[4]}$

The quality of root canal fillings is associated with ideal biomechanical instrumentation and followed by homogeneity obturation of the root canal structure. ${ }^{[5,6]}$ On the other hand, the type of roots and the morphology characteristics of mandibular molar teeth present clinical complications thatoftenjeopardize theendodontic therapy. Therefore, clinicians must be able to identify the root canal structure before or during a root canal treatment. ${ }^{[6]}$ It is known that the presence of additional canals or deviations of the main root canals can cause endodontic flare-ups and failures. ${ }^{[7-9]}$ Successful treatment of endodontic complications is associated with diagnostic imaging techniques that provide information about the teeth and

\footnotetext{
How to cite this article: Nur BG, Ok E, Altunsoy M, Aglarci OS, Colak M, Gungor E. Evaluation of the root and canal morphology of mandibular permanent molars in a south-eastern Turkish population using cone-beam computed tomography. Eur J Dent 2014;8:154-9. 
their surrounding structures. ${ }^{[10]}$ These techniques include panoramic, ${ }^{[11,12]}$ full-mouth periapical radiograph, ${ }^{[5,13,14]}$ and cone-beam computed tomography (CBCT). ${ }^{[15,16]}$ Conventional radiograph images are widely used in endodontic treatment, but they are not very beneficial in the assessment of complex root canal anatomies where anatomic structures may be confused with periapical pathosis. ${ }^{[7,15]}$ Recently, the use of three-dimensional (3D) imaging techniques such as $\mathrm{CBCT}$ provides much more detail about the root canal morphology and is more sensitive to detection of supplemental canals than are radiographic images. ${ }^{[15,16]}$ Studies suggest that CBCT potentially provides the clinician a more accurate assessment of the outcome of root canal treatments. ${ }^{[6,17]}$ Recently, some studies have shown that CBCT scanning is able to evaluate the morphology of root canals. ${ }^{[6,17,18]} \mathrm{A}$ review of the literature on root and canal morphology using the PubMed database identified several reports on the root and canal morphology with different techniques in a Turkish population. ${ }^{[3,6,19,20]}$ However, there is one study that evaluated root and canal morphology of the mandibular molars in a Turkish population by using CBCT. ${ }^{[6]}$

The aim of this retrospective study was to analyze the root and canal morphology of mandibular molar teeth in a Turkish population from the south-eastern region of Turkey by using CBCT images.

\section{MATERIALS AND METHODS}

CBCT images of mandibular molars were obtained from patients who visited the Faculty of Dentistry, Dicle University, Diyarbakir, Turkey, for various purposes between May 2009 and April 2012. All records were selected from a Turkish population from the south-eastern region of Turkey. CBCT images of 421 women and 429 men between the ages of 14 and 70 years were examined. In total, 2131 teeth (966 first mandibular molars and 1165 second mandibular molars) were evaluated in this study. This study was based on the retrospective evaluation of $\mathrm{CBCT}$, and approved by the Medical Ethics Committee of Sifa University (Protocol \#33-2013).

The following were the inclusion criteria for selecting the teeth:

a. Permanent molars with no periapical lesions

b. No root canals with open apices, resorption, or calcification

c. Fully erupted teeth

d. No root canal fillings, posts, and crown restorations

e. CBCT images of good quality.
The exclusion criteria included the following:

a. Image deformity on CBCT

b. Incomplete root formation

c. Permanent mandibular third molars that had shifted mesially to the position of early lost second molars were identified by tooth morphology and tilting and were excluded.

The CBCT images were obtained using a CBCT scanner (I-CAT Vision TM; Imaging Science International, Hatfield, PA, USA, 2008) at $120 \mathrm{kVp}$, $18.54 \mathrm{~mA}$ with an exposure time of $8-9 \mathrm{~s}$. The voxel size of the images was $0.3 \mathrm{~mm}$. An experienced radiologist acquired the images according to the manufacturer's instructions. Intra-examiner calibration of the CBCT images was first performed to evaluate the reliability of the assessment. All the images were assessed separately twice by two examiners (one endodontist and one maxillofacial radiologist) with a 2-week interval between the assessments. The specimens were investigated radiographically by $\mathrm{CBCT}$ and the following were observed:

a. The number of roots

b. The number of canals per root

c. The canal configuration in each root using Vertucci's classification ${ }^{[21]}$

d. The frequency of additional roots

e. Differences in the teeth of males and females.

Statistically significant differences were evaluated using the Chi-square test with SPSS (Version 17.0; SPSS Inc., Chicago, IL, USA) software, and $P<0.05$ were considered as statistically significant.

\section{RESULTS}

The results of this study are summarized in Tables 1 and 2, and variants in the root canal morphology of the mandibular first and second permanent molars are shown in Figure 1.

\section{Mandibular first molars}

The majority of first molars (99.2\%) had one mesial and one distal root. Only five patients (three females and two males) were detected as having three-rooted $(0.5 \%)$ molars and three patients (all females) were detected as having one-rooted $(0.3 \%)$ molars. The three-rooted molars had distolingual or mesiolingual roots and all additional roots had a type I configuration. There was no statistical difference related to gender $(P>0.05)$ in the number of roots of mandibular first molars. 
The frequency and distribution of all root canals are listed in Table 1 . In total, $96.8 \%$ of the mesial roots had two canals, $3 \%$ had one canal, and $0.2 \%$ had three canals. In the distal root, $49.8 \%$ had two canals, $49.8 \%$ had one canal, and $0.4 \%$ had three canals.

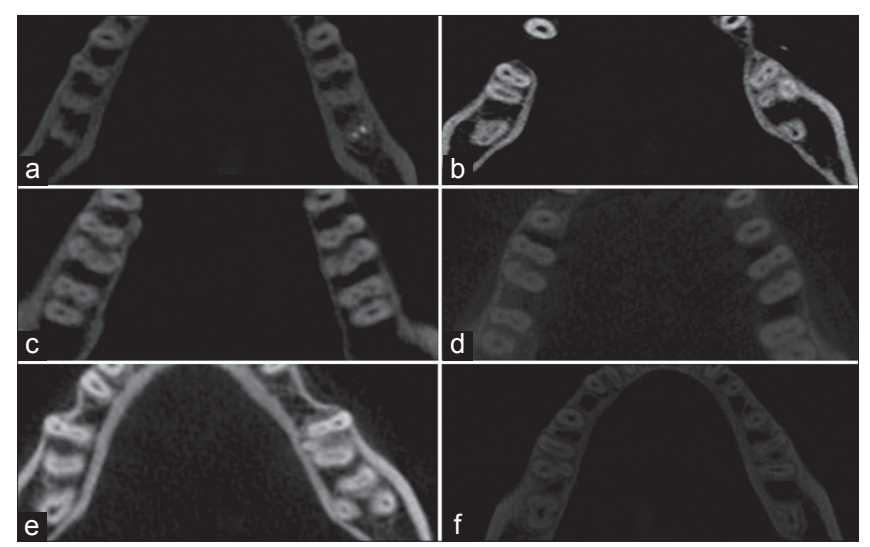

Figure 1: (a) Mandibular bilateral first molars with two roots and four canals. (b) Mandibular left first molar with two roots, right first molar with three roots. (c) Bilateral mandibular first molars with five canals and three roots. (d) Mandibular bilateral first molars with two roots and four canals. (e) Mandibular right first molars with three roots and four canals, mandibular right second molar with three roots and three canals, and left second molar with one root and one canal. (f) Mandibular right first molars with two roots and two canals and left first molars with three roots and three canals
There were six variants in the root canal morphology of the mandibular first molars. The distribution and percentages of the six categories of variants in the root canal anatomy of the mandibular first molars are listed in Table 2. Type IV canal configuration was the most prevalent in the mesial roots, whereas type I canal configuration was the most prevalent in the distal roots. Additionally, type II, type III, type V, and type VIII canal configurations were observed in mesial and distal roots. Moreover, the incidences of varying root canal configurations did not statistically differ between females and males $(P>0.05)$.

\section{Mandibular second molars}

Most of the second molars (90\%) had one mesial and one distal root, whereas $10 \%$ had one root. No statistical gender-related difference $(P>0.05)$ was detected in the incidence of root numbers of mandibular second molars.

The incidences of root canals are listed in Table 1. In total, $90 \%$ of the mesial roots had two canals and $10 \%$ had one canal. The majority of distal roots had one canal $(97 \%)$ and $3 \%$ had two canals. Additionally, three patients $(0.01 \%)$ had additional (distolingual or

\begin{tabular}{|c|c|c|c|c|c|c|c|c|c|c|c|c|}
\hline \multirow{3}{*}{$\begin{array}{l}\text { Variables } \\
\text { Number of canals }\end{array}$} & \multicolumn{6}{|c|}{ First molar } & \multicolumn{6}{|c|}{ Second molar } \\
\hline & \multicolumn{2}{|c|}{ Mesial root } & \multicolumn{2}{|c|}{ Distal root } & \multicolumn{2}{|c|}{ Extra root } & \multicolumn{2}{|c|}{ Mesial root } & \multicolumn{2}{|c|}{ Distal root } & \multicolumn{2}{|c|}{ Extra root } \\
\hline & $\mathbf{F}$ & M & $\mathbf{F}$ & M & $\mathbf{F}$ & M & $\mathbf{F}$ & M & $\mathbf{F}$ & M & $F$ & M \\
\hline Single canal & $22(4.6)$ & $6(1.3)$ & $167(55)$ & $150(44.7)$ & $3(100)$ & $5(55.6)$ & $70(12)$ & $44(7.6)$ & $553(97.7)$ & $553(96)$ & $1(50)$ & $1(100)$ \\
\hline Two canals & $458(95)$ & $484(98.7)$ & $135(44.4)$ & $185(55.2)$ & 0 & $4(44.4)$ & $512(88)$ & $539(92.4)$ & $13(2.3)$ & $22(4)$ & $1(50)$ & 0 \\
\hline Three canals & $1(0.4)$ & 0 & 0 & 0 & 0 & 0 & 0 & 0 & 0 & 0 & 0 & 0 \\
\hline Four canals & 0 & 0 & 0 & 0 & 0 & 0 & 0 & 0 & 0 & 0 & 0 & 0 \\
\hline Five canals & 0 & 0 & 0 & 0 & 0 & 0 & 0 & 0 & 0 & 0 & 0 & 0 \\
\hline Total & 481 & 490 & 303 & 335 & 3 & 9 & 582 & 583 & 566 & 575 & 2 & 1 \\
\hline
\end{tabular}

\begin{tabular}{|c|c|c|c|c|c|c|c|c|c|c|c|c|}
\hline \multirow{3}{*}{$\begin{array}{l}\text { Variables } \\
\text { Canal configuration }\end{array}$} & \multicolumn{6}{|c|}{ First molar } & \multicolumn{6}{|c|}{ Second molar } \\
\hline & \multicolumn{2}{|c|}{ Mesial root } & \multicolumn{2}{|c|}{ Distal root } & \multicolumn{2}{|c|}{ Extra root } & \multicolumn{2}{|c|}{ Mesial root } & \multicolumn{2}{|c|}{ Distal root } & \multicolumn{2}{|c|}{ Extra root } \\
\hline & $\mathbf{F}$ & M & $\mathbf{F}$ & M & $\mathbf{F}$ & M & $\mathbf{F}$ & M & $\mathbf{F}$ & M & $\mathbf{F}$ & M \\
\hline Type I & $27(5)$ & $25(5)$ & $282(60)$ & $349(59)$ & $3(100)$ & $5(100)$ & $86(15)$ & $59(10)$ & $562(95)$ & $537(93)$ & $1(50)$ & $1(100)$ \\
\hline Type II & $5(1)$ & $5(1)$ & $65(14)$ & $68(11)$ & 0 & 0 & $10(2)$ & $15(2.6)$ & $19(3.2)$ & $20(3)$ & 0 & 0 \\
\hline Type III & $1(0.2)$ & 0 & $9(2)$ & $12(2)$ & 0 & 0 & $1(0.2)$ & $7(1.2)$ & 0 & 0 & 0 & 0 \\
\hline Type IV & $428(89)$ & $455(93)$ & $95(20)$ & $110(19)$ & 0 & 0 & $440(76)$ & $487(84)$ & $10(1.7)$ & $17(2.9)$ & $1(50)$ & 0 \\
\hline Type V & $14(3)$ & $3(0.6)$ & $20(4)$ & $50(8)$ & 0 & 0 & $45(8)$ & $15(2.6)$ & $2(0.3)$ & $1(0.2)$ & 0 & 0 \\
\hline Type VI & 0 & 0 & 0 & 0 & 0 & 0 & 0 & 0 & 0 & 0 & 0 & 0 \\
\hline Type VII & 0 & 0 & 0 & 0 & 0 & 0 & 0 & 0 & 0 & 0 & 0 & 0 \\
\hline Type VIII & $1(0.2)$ & 0 & 0 & 0 & 0 & 0 & 0 & 0 & 0 & 0 & 0 & 0 \\
\hline Total & 476 & 488 & 471 & 589 & 3 & 5 & 582 & 583 & 593 & 575 & 2 & 1 \\
\hline
\end{tabular}


mesiolingual) canals. The frequency distribution of the number of root canals did not differ between females and males $(P>0.05)$.

There were five variants in the root canal morphology of the mandibular second molars. The distribution and percentages of the five categories of variants in the root canal anatomy of the mandibular second molars are listed in Table 2. Type IV canal configuration was the most prevalent in the mesial roots, whereas type I canal configuration was the most prevalent in the distal roots. Additionally, type II, type III, and type V canal configurations were also observed in mesial and distal roots, and the incidences of varying root canal configurations did not statistically differ between females and males $(P>0.05)$, with the exception being the mesial canal of the left mandibular second molars $(P<0.05)$.

\section{DISCUSSION}

It is essential to have a thorough knowledge of root canal morphology and configuration for successful endodontic treatment. ${ }^{[4]}$ The failure to determine additional canals and incomplete instrumentation are the most likely causes of endodontic flare-ups and failures. ${ }^{[-9]}$ Many studies have examined root and canal morphology using various methods such as canal staining and clearing technique, ${ }^{[4,22]}$ cross-sectioning technique, ${ }^{[23]}$ contrast medium-enhanced radiography, ${ }^{[24]}$ modified canal staining and clearing, ${ }^{[25]}$ radiographic examination, ${ }^{[26]}$ and computed tomography scanning. ${ }^{[27,28]}$ However, canal staining and clearing technique ${ }^{[4,22]}$ and cross-sectioning technique ${ }^{[23]}$ are invasive and result in irreversible damage to samples. CBCT is a non-invasive method compared with cross-sections and the clearing technique, and can be used directly to evaluate patients. ${ }^{[17]}$ Conventional intraoral periapical radiographs provide only two-dimensional images, and in some cases, anatomic structures can be superimposed in these images; therefore, they are not beneficial in the evaluation of complex root canal anatomies due to their natural limitations. ${ }^{[28]}$

CBCT has been widely used to evaluate the endodontic applications by clinicians in the past few years, and provides clinicians with three-dimensional information about the external and internal morphology of the root and canal systems. ${ }^{[6,17,29,30]}$ In the present study, CBCT provides a comprehensive report on the root canal morphology of mandibular first and second molars in a Turkish population.
It has usually been accepted that mandibular first molars have two roots located mesially and distally and three canals (one root canal in the distal root and two root canals located in the mesial root); but in populations with Mongoloid traits, the additional root in a mandibular first molar is considered to be a normal morphologic version and may be defined as a Mongolian trait or an Asian trait. ${ }^{[31]}$ It has been described that the Mongoloid population exhibits significantly more mandibular first molars with three roots (3:1) than the other populations, and this version could be considered a genetically determined characteristic. ${ }^{[32]}$ In this study, we found that $0.5 \%$ of mandibular first molars and $0.01 \%$ of mandibular second molars had an additional root located distolingually. This frequency is less than that reported in a previous study of Turkish population $(2.06 \%),{ }^{[6]}$ but is very less than that reported in Japanese $(22.7 \%),{ }^{[33]}$ Koreans $(22.3 \%),{ }^{[32]}$ Hong Kong population $(15.0 \%),{ }^{[34]}$ and by Zhang et al. $(29 \%)^{[35]}$ who reported a high prevalence of three roots in an Asian population. Furthermore, this variant has a frequency of lower than 5\% in European people (British, Dutch, German, Finnish), African (Bushmen, Bantu, Senegalese) and Indian populations. ${ }^{[21,36]}$

In the case of mandibular second molars, the majority (90\%; 1051 teeth) had two roots located mesially and distally and 114 teeth $(10 \%)$ had one root. The incidence of two separate roots is similar to that reported in a previous study of Turkish population by Demirbuga et al. $(85.4 \%){ }^{[6]}$ however, it is higher than that reported in Burmese $(58.2 \%)^{[37]}$ and Thai $(54 \%)$ populations. ${ }^{[36]}$ In the present study, the frequency of two canals in the mesial and distal roots of the first molar tooth was $96.8 \%$ and $49.8 \%$, respectively, whereas the second molar tooth had two canals in $90.2 \%$ and $3.15 \%$ of the mesial and distal roots, respectively. This incidence is similar to those reported in the previous studies of Turkish and Western Chinese populations by using CBCT, except for the distal roots of the first molar tooth. ${ }^{[6,38]}$

In the present study, type IV configuration was the most prevalent $(89 \%$ in females and $93 \%$ in males) in mesial roots. This is consistent with the findings of many earlier studies. ${ }^{[4,6,20,36-38]}$ However, Zaatar et al. ${ }^{[39]}$ and al-Nazhan ${ }^{[40]}$ reported type II being the most prevalent followed by type IV. Only one patient $(0.2 \%$ of females) had a type VIII configuration in the mesial root, which is in agreement with the study by Chen et al. $(0.2-5 \%) .{ }^{[37]}$ The most common canal configuration of distal roots was type I, with a reported frequency between 
$54 \%$ and $72 \% .{ }^{[6,20,21,32,36,40]}$ Likewise, in our study, type I configuration was the most prevalent in females $(60 \%)$ and males $(59 \%)$ in the distal roots. Furthermore, in this study, type II, type IV, and type $\mathrm{V}$ configurations were found to be higher than that reported by Demirbuga et al. ${ }^{[6]} \mathrm{Gu}$ et al. ${ }^{[41]}$ examined 20 extracted three-rooted mandibular first molars in a micro-computed tomography study of Chinese patients. They found that all the additional roots contained a type I (100\%) canal configuration. In the present study, all the additional roots contained a type I canal configuration The results of the present investigation are in agreement with previous studies. ${ }^{[3,4,6,37]}$

The most common root canal configuration of second molar teeth in the present study was type IV $(76 \%$ for females and $84 \%$ for males) in the mesial roots and type I (95\% for females and 93\% for males) in the distal roots [Table 2]. This finding agrees with those of Demirbuga et al. ${ }^{[6]}$ and Ahmed et al., ${ }^{[42]}$ but contrasts with those of Gulabivala et al., ${ }^{[36]}$ Vertucci and Williams, ${ }^{[21]}$ and Pineda and Kuttler ${ }^{[26]}$ who observed type I as the most frequent canal configuration in the mesial root of the second molar tooth in a Caucasian population.

Several possible reasons may account for the differences, such as ethnic population, study design (the samples in the other studies were extracted teeth), ${ }^{[21]}$ and the sample size. These variables may result in different frequencies of configuration of the mandibular molars.

\section{CONCLUSIONS}

The root number and morphology of 850 Turkish mandibular molars were examined using CBCT. In the present study, type IV and type I canal configurations were the most prevalent in the mesial and distal roots, respectively, of both the mandibular first and second molar teeth. There was a low prevalence of three-rooted mandibular molars in this Turkish population. CBCT scanning provides supplemental information about the root canal configurations of mandibular molars in a Turkish population, and this study may help clinicians in the root canal treatment of mandibular molars.

\section{ACKNOWLEDGMENTS}

The authors deny any conflicts of interest. They affirm that they have no financial affiliation (e.g. employment, direct payment, stock holdings, retainers, consultantship, patent licensing arrangements, or honoraria) or involvement with any commercial organization with direct financial interest in the subject or materials discussed in this manuscript. Nor did they have any such arrangement in the past 3 years.

\section{REFERENCES}

1. Trope M, Elfenbein L, Tronstad L. Mandibular premolars with more than one root canal in different race groups. J Endod 1986;12:343-5.

2. Awawdeh L, Abdullah H, Al-Qudah A. Root form and canal morphology of Jordanian maxillary first premolars. J Endod 2008;34:956-61.

3. Sert S, Bayirli GS. Evaluation of the root canal configurations of the mandibular and maxillary permanent teeth by gender in the Turkish population. J Endod 2004;30:391-8.

4. Vertucci FJ. Root canal anatomy of the human permanent teeth. Oral Surg Oral Med Oral Pathol 1984;58:589-99.

5. Unal GC, Kececi AD, Kaya BU, Tac AG. Quality of root canal fillings performed by undergraduate dental students. Eur J Dent 2011;5:324-30.

6. Demirbuga S, Sekerci AE, Dinçer AN, Cayabatmaz M, Zorba YO. Use of cone-beam computed tomography to evaluate root and canal morphology of mandibular first and second molars in Turkish individuals. Med Oral Patol Oral Cir Bucal 2013;18:e737-44.

7. Slowey RR. Root canal anatomy. Road map to successful endodontics. Dent Clin North Am 1979;23:555-73.

8. England MC Jr, Hartwell GR, Lance JR. Detection and treatment of multiple canals in mandibular premolars. J Endod 1991;17:174-8.

9. Barbizam JV, Ribeiro RG, Tanomaru Filho M. Unusual anatomy of permanent maxillary molars. J Endod 2004;30:668-71.

10. Liang YH, Yuan M, Li G, Shemesh H, Wesselink PR, Wu MK. The ability of cone-beam computed tomography to detect simulated buccal and lingual recesses in root canals. Int Endod J 2012;45:724-9.

11. De Moor RJ, Hommez GM, De Boever JG, Delmé KI, Martens GE. Periapical health related to the quality of root canal treatment in a Belgian population. Int Endod J 2000;33:113-20.

12. Kabak Y, Abbott PV. Prevalence of apical periodontitis and the quality of endodontic treatment in an adult Belarusian population. Int Endod J 2005;38:238-45.

13. Adebayo ET, Ahaji LE, Nnachetta RN, Nwankwo O, Akabogu-Okpeseyi N, Yaya MO, et al. Technical quality of root canal fillings done in a Nigerian general dental clinic. BMC Oral Health 2012;12:42.

14. Gündüz K, Avsever H, Orhan K, Demirkaya K. Cross-sectional evaluation of the periapical status as related to quality of root canal fillings and coronal restorations in a rural adult male population of Turkey. BMC Oral Health 2011;11:20.

15. D'Addazio PS, Campos CN, Özcan M, Teixeira HG, Passoni RM, Carvalho AC. A comparative study between cone-beam computed tomography and periapical radiographs in the diagnosis of simulated endodontic complications. Int Endod J 2011;44:218-24.

16. Durack C, Patel S. Cone beam computed tomography in endodontics. Braz Dent J 2012;23:179-91.

17. Yang H, Tian C, Li G, Yang L, Han X, Wang Y. A cone-beam computed tomography study of the root canal morphology of mandibular first premolars and the location of root canal orifices and apical foramina in a Chinese subpopulation. J Endod 2013;39:435-8.

18. Silva EJ, Nejaim Y, Silva AV, Haiter-Neto F, Cohenca N. Evaluation of root canal configuration of mandibular molars in a Brazilian population by using cone-beam computed tomography: An in vivo study. J Endod 2013;39:849-52.

19. Sert S, Aslanalp V, Tanalp J. Investigation of the root canal configurations of mandibular permanent teeth in the Turkish population. Int Endod J 2004;37:494-9.

20. Calişkan MK, Pehlivan Y, Sepetçioğlu F, Türkün M, Tuncer SS. Root canal morphology of human permanent teeth in a Turkish population. J Endod 1995;21:200-4.

21. Vertucci FJ, Williams RG. Root canal anatomy of the mandibular first molar. J N J Dent Assoc 1974;45:27-8 passim. 
22. Al-Qudah AA, Awawdeh LA. Root and canal morphology of mandibular first and second molar teeth in a Jordanian population. Int Endod J 2009;42:775-84.

23. Weine FS, Healey HJ, Gerstein H, Evanson L. Canal configuration in the mesiobuccal root of the maxillary first molar and its endodontic significance. Oral Surg Oral Med Oral Pathol 1969;28:419-25.

24. Fan B, Gao Y, Fan W, Gutmann JL. Identification of a C-shaped canal system in mandibular second molars-part II: The effect of bone image superimposition and intraradicular contrast medium on radiograph interpretation. J Endod 2008;34:160-5.

25. Weng XL, Yu SB, Zhao SL, Wang HG, Mu T, Tang RY, et al. Root canal morphology of permanent maxillary teeth in the Han nationality in Chinese Guanzhong area: A new modified root canal staining technique. J Endod 2009;35:651-6.

26. Pineda F, Kuttler Y. Mesiodistal and buccolingual roentgenographic investigation of 7,275 root canals. Oral Surg Oral Med Oral Pathol 1972;33:101-10.

27. Zheng QH, Wang Y, Zhou XD, Wang Q, Zheng GN, Huang DM. A cone-beam computed tomography study of maxillary first permanent molar root and canal morphology in a Chinese population. J Endod 2010;36:1480-4.

28. Purra AR, Mushtaq M, Robbani I, Farooq R. Spiral computed tomographic evaluation and endodontic management of a mandibular second molar with four roots. A case report and literature review. Iran Endod J 2013;8:69-71.

29. Cotton TP, Geisler TM, Holden DT, Schwartz SA, Schindler WG. Endodontic applications of cone-beam volumetric tomography. J Endod 2007;33:1121-32.

30. Scarfe WC, Levin MD, Gane D, Farman AG. Use of cone beam computed tomography in endodontics. Int J Dent 2009;2009:634567.

31. de Pablo OV, Estevez R, Péix Sánchez M, Heilborn C, Cohenca N. Root anatomy and canal configuration of the permanent mandibular first molar: A systematic review. J Endod 2010;36:1919-31.

32. Park JB, Kim N, Park S, Kim Y, Ko Y. Evaluation of root anatomy of permanent mandibular premolars and molars in a Korean population with cone-beam computed tomography. Eur J Dent 2013;7:94-101.

33. de Souza-Freitas JA, Lopes ES, Casati-Alvares L. Anatomic variations of lower first permanent molar roots in two ethnic groups. Oral Surg Oral Med Oral Pathol 1971;31:274-8.
34. Fabra-Campos H. Unusual root anatomy of mandibular first molars J Endod 1985;11:568-72.

35. Zhang $\mathrm{R}$, Wang $\mathrm{H}$, Tian $\mathrm{YY}, \mathrm{Yu} \mathrm{X}, \mathrm{Hu} \mathrm{T}$, Dummer PM. Use of cone-beam computed tomography to evaluate root and canal morphology of mandibular molars in Chinese individuals. Int Endod J 2011;44:990-9.

36. Gulabivala K, Opasanon A, Ng YL, Alavi A. Root and canal morphology of Thai mandibular molars. Int Endod J 2002;35:56-62.

37. Chen YC, Lee YY, Pai SF, Yang SF. The morphologic characteristics of the distolingual roots of mandibular first molars in a Taiwanese population. J Endod 2009;35:643-5.

38. Wang Y, Zheng QH, Zhou XD, Tang L, Wang Q, Zheng GN, et al. Evaluation of the root and canal morphology of mandibular first permanent molars in a western Chinese population by cone-beam computed tomography. J Endod 2010;36:1786-9.

39. Zaatar EI, al Anizi SA, al Duwairi Y. A study of the dental pulp cavity of mandibular first permanent molars in the Kuwaiti population. J Endod 1998;24:125-7.

40. al-Nazhan S. Incidence of four canals in root-canal-treated mandibular first molars in a Saudi Arabian sub-population. Int Endod J 1999;32:49-52.

41. Gu Y, Lu Q, Wang H, Ding Y, Wang P, Ni L. Root canal morphology of permanent three-rooted mandibular first molars-part I: Pulp floor and root canal system. J Endod 2010;36:990-4.

42. Ahmed HA, Abu-bakr NH, Yahia NA, Ibrahim YE. Root and canal morphology of permanent mandibular molars in a Sudanese population. Int Endod J 2007;40:766-71.

\begin{tabular}{|l|l|}
\hline \multicolumn{2}{|c|}{ Access this article online } \\
\hline Quick Response Code: & Website: \\
& www.eurjent.com \\
& \\
&
\end{tabular}

